\title{
PERIODIC TRANSFORMATIONS ON THE PRODUCT OF TWO SPHERES
}

BY

J. C. SU(1)

1. Introduction. This paper is concerned with a transformation group $\left(Z_{p} ; X\right)$, where $Z_{p}$ is a cyclic group of prime order $p$ and $X$ is a space having the same $\bmod p$ cohomology algebra of $S^{m} \times S^{n}$, the product of an $m$-sphere and an $n$-sphere. This problem has the obvious motivation. If we have a pair $\left(Z_{p} ; X_{1}\right)$ and $\left(Z_{p} ; X_{2}\right)$ of transformation groups, where $X_{1}$ is a $\bmod p$ cohomology $m$ sphere and $X_{2}$ a $\bmod p$ cohomology $n$-sphere, then the diagonal action $\left(Z_{p} ; X_{1} \times X_{2}\right)$ is a transformation group of the type just mentioned. According to the well-known theorem of P. A. Smith [4], the fixed point set has the same $\bmod p$ cohomology algebra of the product of two spheres. That is, the fixed point set inherits the same cohomology characteristics of the space. For the general case, there is a theorem of R. G. Swan [7] in which sufficient conditions are given to insure that the fixed point set does have the $\bmod p$ cohomology algebra of the product of two spheres. Following the same technique devised by him, we succeeded in refining his result to the extent that the cohomology algebra of the fixed point set is entirely determined. The process is a rather tedious case by case study. To state briefly, there are a variety of possibilities for the cohomology of components of the fixed point set. The components can be like a point, a sphere, a product of two spheres, a projective space, or a Klein bottle. To justify the unpleasant nature of this long list, we give some examples showing that most of the cases do happen. Moreover, these examples are all nice differentiable actions on manifolds. Thus in a sense there is not much room left for further improvement. Finally, our result also indicates there is still something of the space that is passed over to the fixed point set. Namely the Poincare duality is valid for every component of the fixed point set. Whether this is true in general might be an interesting conjecture.

The author is indebted to Professor E. E. Floyd for his help, particularly in the construction of examples, and to Professor L. N. Mann for bringing this problem to my attention.

2. The spectral sequence of R. G. Swan. Let $\left(Z_{p} ; X\right)$ be a transformation group of $Z_{p}$ on a compact Hausdorff space $X$ with fixed point set $F$ (possibly

Presented to the Society, April 29, 1963 ; received by the editors April 29, 1963.

(1) This research was supported by the Air Force Office of Scientific Research. 
empty). $H^{*}\left(X ; Z_{p}\right)=\Sigma_{0}^{\infty} H^{k}\left(X ; Z_{p}\right)\left(H^{*}\left(F ; Z_{p}\right)=\Sigma_{0}^{\infty} H^{k}\left(F ; Z_{p}\right)\right)$ denotes the (nonreduced) Alexander-Wallace-Spanier cohomology algebra of $X$ (of $F$ ) with coefficients in $Z_{p}$.

Let $A^{*}\left(X ; Z_{p}\right)=\Sigma_{0} A^{k}\left(X ; Z_{p}\right)\left(A^{*}\left(F ; Z_{p}\right)=\Sigma_{0}^{\infty} A^{k}\left(F ; Z_{p}\right)\right)$ be the AWS cochain group of $X$ (of $F$ ) with coefficients in $Z_{p}$. They are $Z_{p}$-modules in a natural way. Let $W^{*}=\Sigma_{-\infty}^{\infty} W^{k}$ be a complete resolution of $Z_{p}$ [2, Chapter XII, §3]. Consider the double complexes $\operatorname{Hom}_{Z_{p}}\left(W^{*} ; A^{*}\left(X ; Z_{p}\right)\right)=\sum_{i j} \operatorname{Hom}_{z_{p}}\left(W^{i} ; A^{j}\left(X ; Z_{p}\right)\right)$ and $\operatorname{Hom}_{Z_{p}}\left(W^{*} ; A^{*}\left(F ; Z_{p}\right)\right)=\Sigma_{i j} \operatorname{Hom}_{Z_{p}}\left(W^{i} ; A^{j}\left(F ; Z_{p}\right)\right.$ ) (for the notation $\operatorname{Hom}_{Z_{p}}(;)$, see [2, Chapter XII]). The first filtration [2, Chapter XV, §6] of these complexes gives rise to two convergent spectral sequences [2, Chapter $\mathrm{XV}, \S 3] E_{r}(X)$ and $E_{r}(F)$. According to R. G. Swan [7], the main properties of these spectral sequences are as follows:

$$
\begin{aligned}
& E_{2}^{s, t}(X)=\hat{H}^{s}\left(Z_{p} ; H^{t}\left(X ; Z_{p}\right)\right), \\
& E_{2}^{s, t}(F)=\hat{H}^{s}\left(Z_{p} ; H^{t}\left(F ; Z_{p}\right)\right),-\infty<s<\infty, 0 \leqq t,
\end{aligned}
$$

where $\hat{H}^{s}\left(Z_{p} ; A\right)$ is the $s$-dimensional Tate cohomology group [2, Chapter XII] of $Z_{p}$ with coefficients in a $Z_{p}$-module $A$.

(2.2) $E_{\infty}(X)$ is associated with $J^{*}(X)=H^{*}\left(\operatorname{Hom}_{Z_{p}}\left(W^{*} ; A^{*}\left(X ; Z_{p}\right)\right)\right.$ and $E_{\infty}(F)$ is associated with $J^{*}(F)=H^{*}\left(\operatorname{Hom}_{Z_{p}}\left(W^{*} ; A^{*}\left(F ; Z_{p}\right)\right)\right.$.

(2.3) The spectral sequence $E_{r}(F)$ is always trivial and $E_{2}(F)$ is canonically isomorphic to $J^{*}(F)$ as graded algebra. Notice that $Z_{p}$ acts trivially on $H^{*}\left(F ; Z_{p}\right)$, hence

$$
E_{2}(F)=\hat{H}^{*}\left(Z_{p} ; Z_{p}\right) \otimes H^{*}\left(F ; Z_{p}\right) .
$$

Now the inclusion $i: F \rightarrow X$ induces an homomorphism $i^{*}: J^{*}(X) \rightarrow J^{*}(F)$. The crucial fact about $i^{*}$ is that

(2.4) $i^{*}: J^{*}(X) \rightarrow J^{*}(F)$ is an isomorphism of graded algebras. It also preserves filtration but is not necessarily an isomorphism with respect to filtration.

In case that $F=\varnothing$, then by convention $E_{2}(F)=J^{*}(F)=0$ in (2.1) through (2.4).

We mention a few more elementary facts. Since $\operatorname{dim} J^{q}(F)=\Sigma_{s} \operatorname{dim} E_{2}^{q-s, s}(F)$ $=\Sigma_{s} \operatorname{dim} \hat{H}^{q-s}\left(Z_{p} ; H^{s}\left(F ; Z_{p}\right)\right)=\Sigma_{s} \operatorname{dim} H^{s}\left(F ; Z_{p}\right)=\operatorname{dim} H^{*}\left(F ; Z_{p}\right)$. By (2.4), we have

$$
\operatorname{dim} H^{*}\left(F ; Z_{p}\right)=\operatorname{dim} J^{q}(X) \text { for all } q .
$$

As usual, the filtration of $J^{q}(X)$ is denoted by

$$
\cdots \supset J^{q-s, s}(X) \supset J^{q-s+1, s-1}(X) \supset \cdots \supset J^{q, 0}(X) \supset 0
$$

with $J^{q-s, s}(X) / J^{q-s+1, s-1}(X)=E_{\infty}^{q-s, s}(X)$. Since $i^{*}\left(J^{q-s+1, s-1}(X)\right) \subset J^{q-s+1, s-1}(F)$. we have an epimorphism

$$
J^{q}(X) / J^{q-s+1, s-1}(X) \rightarrow J^{q}(F) / J^{q-s+1, s-1}(F)
$$

for all $q$ and $s$. Computing the dimensions for both terms yields 


$$
\begin{aligned}
\sum_{k \geqq s} \operatorname{dim} H^{k}\left(F ; Z_{p}\right) & \leqq \sum_{k \geqq s} \operatorname{dim} E_{\infty}^{q-k, k}(X) \leqq \sum_{k \geqq s} \operatorname{dim} E_{2}^{q-k, k}(X) \\
& \leqq \sum_{k \geqq s} \operatorname{dim} H^{k}\left(X ; Z_{p}\right)
\end{aligned}
$$

for all $q$ and $s$. Notice that if $\Sigma_{k \geqq 1} \operatorname{dim} H^{k}\left(F ; Z_{p}\right)=\Sigma_{k \geqq 1} \operatorname{dim} E_{\infty}^{q-k, k}(X)$ for some $q$, then $J^{q}(X) / J^{q, 0}(X) \rightarrow J^{q}(F) / J^{q, 0}(F)$ is an isomorphism. Therefore we have

$$
\begin{aligned}
i^{*}: J^{q, 0}(X) \rightarrow J^{q, 0}(F) & \text { is an isomorphism if } \sum_{k \geqq 1} \operatorname{dim} H^{k}\left(F ; Z_{p}\right) \\
& =\sum_{k \geqq 1} \operatorname{dim} E_{\infty}^{q-k, k}(X) .
\end{aligned}
$$

Finally, let $\chi_{p}(X)$ and $\chi_{p}(F)$ be the $\bmod p$ Euler characteristic of $X$ and $F$ respectively. We have the relation of E. E. Floyd [3]

$$
\chi_{p}(F) \equiv \chi_{p}(X) \bmod p,
$$

where by convention, $\chi_{p}(F)=0$ if $F$ is empty.

Now for the rest of this paper, we assume that $H^{*}\left(X ; Z_{p}\right)=H^{*}\left(S^{m} \times S^{n} ; Z_{p}\right)$. That is, $H^{*}\left(X ; Z_{p}\right)=\wedge[a] \otimes \wedge[b]$ is the tensor product of exterior algebras over $Z_{p}$ with generators $a$ and $b$ of degrees $m$ and $n$ respectively. We assume that $0<m \leqq n$ since the case $m=0$ is clearly of no interest. We shall write $a b$ in place of $a \otimes b$ and use $1 \in H^{0}\left(X ; Z_{p}\right)$ to denote the unit of $H^{*}\left(X ; Z_{p}\right)$.

3. The case when $Z_{p}$ acts trivially on $H^{*}\left(X ; Z_{p}\right)$. Throughout this section, we assume that $Z_{p}$ acts trivially on $H^{*}\left(X ; Z_{p}\right)$. This is the case, for example, if $m \neq n$. By the Künneth formula, we have

$$
E_{2}(X)=\hat{H}^{*}\left(Z_{p} ; Z_{p}\right) \otimes H^{*}\left(X ; Z_{p}\right) .
$$

The algebra $\hat{H}^{*}\left(Z_{p} ; Z_{p}\right)$ can be described as follows. Additively, $H^{s}\left(Z_{p} ; Z_{p}\right)=Z$ for all $s$. Let $t^{s} \in H^{s}\left(Z_{p} ; Z_{p}\right)$ be the generator. If $p=2$, the multiplication is simply given by $t^{s} t^{s^{\prime}}=t^{s+s^{\prime}}$. If $p \neq 2$, the multiplication is given by $t^{s} t^{s^{\prime}}=t^{s+s^{\prime}}$ when $s^{\prime}$ is even and $t^{s} t^{s^{\prime}}=0$ when both $s$ and $s^{\prime}$ are odd [2, Chapter XII, §7]. In any event, $t^{0} \in H^{0}\left(Z_{p} ; Z_{p}\right)$ is a unit and will be denoted by 1 . The multiplication in $E_{2}(X)$ is given by $\left(t^{s} \otimes \alpha\right)\left(t^{s^{\prime}} \otimes \beta\right)=(-1)^{t s^{\prime}}\left(t^{s} t^{s^{\prime}} \otimes \alpha \beta\right)$ for $t^{s} \otimes \alpha \in E_{2}^{s, t}(X)$.

Proposition 3.1. If $p \neq 2$ and $m, n$ are both even, then the spectral sequence $E_{r}(X)$ is trivial.

Proof. We prove by induction that the differential $d_{r}$ on $E_{r}(X)$ is trivial for all $r \geqq 2$. For $r=2$, this is evident. Suppose $d_{s}=0$ for $2 \leqq s<r$, we may identify $E_{2}(X)$ with $E_{r}(X)$ and it suffices to show that $d_{r}(1 \otimes a)=d_{r}(1 \otimes b)=0$. Now $d_{r}\left(E_{r}^{0, m}(X)\right) \subset E_{r}^{r, m-r+1}(X)$. If $d_{r}(1 \otimes a) \neq 0$, we must have $r=m+1$ and we may set $d_{r}(1 \otimes a)=t^{m+1} \otimes 1$. But $(1 \otimes a)(1 \otimes a)=(-1)^{m} 1 \otimes a^{2}=0$, hence 
$0=d_{r}[(1 \otimes a)(1 \otimes a)]=\left(t^{m+1} \otimes 1\right)(1 \otimes a)+(-1)^{m}(1 \otimes a)\left(t^{m+1} \otimes 1\right)=2\left(t^{m+1} \otimes a\right)$. This is a contradiction since $p \neq 2$. In a similar fashion, we also have $d_{r}(1 \otimes b)=0$.

We shall first deal with the case when the spectral sequence $E_{r}(X)$ is nontrivial. If $p=2$, this is very simple.

THEOREM 3.2. Suppose that $p=2$, that $Z_{2}$ acts trivially on $H^{*}\left(X ; Z_{2}\right)$ and that the spectral sequence $E_{r}(X)$ is nontrivial. Then $H^{*}\left(F ; Z_{2}\right)=H^{*}\left(S^{r} ; Z_{2}\right)$ for some $-1 \leqq r \leqq m+n$, where as usual $S^{-1}$ stands for the empty set.

Proof. If $E_{r}(X)$ is nontrivial, then $\operatorname{dim} J^{q}(X)<\Sigma_{i} \operatorname{dim} E_{2}^{q-i, i}(X)$ $=\operatorname{dim} H^{*}\left(X ; Z_{p}\right)=4$. Hence $\operatorname{dim} H^{*}\left(F ; Z_{p}\right) \leqq 3$. If $p=2$, the Euler characteristic relation (2.8) implies that $\operatorname{dim} H^{*}\left(F ; Z_{2}\right)=0$, or 2 . That is, $H^{*}\left(F ; Z_{2}\right)=H^{*}\left(S^{r} ; Z_{2}\right)$. That $r \leqq m+n$ follows from (2.6).

Notice that the multiplication of $H^{*}\left(X ; Z_{2}\right)$ is not used in the argument. Hence (3.2) is true even if $H^{*}\left(X ; Z_{2}\right)=H^{*}\left(S^{m} \times S^{n} ; Z_{2}\right)$ only as a module.

Assume now $p \neq 2$. Let $r \geqq 2$ be the smallest integer such that $d_{r} \neq 0$ on $E_{r}(X)$. Identifying $E_{r}(X)$ with $E_{2}(X)$, we must have either $d_{r}(1 \otimes a) \neq 0$ or $d_{r}(1 \otimes b) \neq 0$.

LEMmA 3.3. If $d_{r}(1 \otimes a) \neq 0$, then $F=\varnothing$.

Proof. Since $d_{r}(1 \otimes a) \in E_{r}^{r, r-m+1}(X)$, we must have $r=m+1$ and we may set $d_{m+1}(1 \otimes a)=t^{m+1} \otimes 1$. Just as in (3.1), one argues that $m$ must be odd. Now $d_{m+1}\left(E_{m+1}^{0, n}(X)\right) \subset E_{m+1}^{m+1, n-m}(X)$, hence we could only have $d_{m+1}(1 \otimes b)=0$, or $n=2 m$ and $d_{m+1}(1 \otimes b)=t^{m+1} \otimes a$, or $n=m$ and $d_{m+1}(1 \otimes b)=t^{m+1} \otimes 1$. Suppose $d_{m+1}(1 \otimes b)=0$. Using the fact that $m+1$ is even, one computes easily that $d_{m+1}\left(t^{s} \otimes a\right)=(-1)^{s}\left(t^{s+m+1} \otimes 1\right) \neq 0$ and $d_{m+1}\left(t^{s} \otimes a b\right)$ $=(-1)^{s}\left(t^{s+m+1} \otimes b\right) \neq 0$. This means $E_{m+1}^{s, m}(X)$ and $E_{m+1}^{s, m+n}(X)$ have no cocycle for all $s$. Similarly $E_{m+1}^{s, n}(X)$ and $E_{m+1}^{s, 0}(X)$ are all coboundary. Thus we have $E_{m+2}(X)=0$ and the assertion follows. Other cases are similar.

Suppose that $d_{r}(1 \otimes a)=0$ and $d_{r}(1 \otimes b) \neq 0$. We have either $r=n+1$ and $d_{n+1}(1 \otimes b)=t^{n+1} \otimes 1$ or $r=n-m+1$ and $d_{n-m+1}(1 \otimes b)=t^{n-m+1} \otimes a$.

LEMMA 3.4. If $r=n+1$ and $d_{n+1}(1 \otimes b)=t^{n+1} \otimes 1$, then $F=\varnothing$.

The proof is completely the same as (3.3).

LEMMA 3.5. If $r=n-m+1, d_{n-m+1}(1 \otimes b)=t^{n-m+1} \otimes a$ and $m$ is even, then $H^{*}\left(F ; Z_{p}\right)=H^{*}\left(S^{r} ; Z_{p}\right)$ for some $-1 \leqq r \leqq m+n$ and $r$ is odd.

Proof. If $m$ is even, $n-m+1$ is even since $n$ must be odd. In this case, $E_{n-m+1}^{s, m}(X)$ is coboundary and $E_{n-m+1}^{s, n}(X)$ has no cocycle for all $s$. Hence $\operatorname{dim} H^{*}\left(F ; Z_{p}\right) \leqq 2$. But $\operatorname{dim} H^{*}\left(F ; Z_{p}\right)=1$ is ruled out by $(2.8)$.

Finally, we must consider the case when $r=n-m+1, d_{n-m+1}(1 \otimes b)=t^{n-m+1}$ $\otimes a$ and both $n$ and $m$ are odd. We seek to eliminate the possibility that $\operatorname{dim} H^{*}(F ; Z)$ 
$=3$. For this purpose, we need the following propositions concerning the functor $\hat{H}^{*}\left(Z_{p} ; A\right)$. Their proof can be found in [5].

Proposition 3.6. If $A \otimes Z_{p}=\operatorname{Tor}\left(A ; Z_{p}\right)=0$, then $\hat{H}^{k}\left(Z_{p} ; A\right)=0$ for all $k$.

Proposition 3.7. If $p \neq 2, A \otimes Z_{p}=Z_{p}$ and $\operatorname{Tor}\left(A ; Z_{p}\right)=0$, then $\hat{H}^{k}\left(Z_{p} ; A\right)=Z_{p}$ for $k$ even and $\hat{H}^{k}\left(Z_{p} ; A\right)=0$ for $k$ odd.

Proposition 3.8. If $p \neq 2, A \otimes Z_{p}=0$ and $\operatorname{Tor}\left(A ; Z_{p}\right)=Z_{p}$, then $\hat{H}^{k}\left(Z_{p} ; A\right)=0$ for $k$ even and $\hat{H}^{k}\left(Z_{p} ; A\right)=Z_{p}$ for $k$ odd.

Notice that (3.8) never occurs if $A$ is a finitely generated abelian group.

Now suppose that $\operatorname{dim} H^{*}\left(F ; Z_{p}\right)=3$. Since $\chi_{p}(X)=0$, we must have $\chi_{p}(F)=3$. According to [7, Corollary 4.2$]$, there is the inequality

$$
\sum_{i \text { even }} \operatorname{dim} H^{i}\left(F ; Z_{p}\right) \leqq \sum_{i \geqq 0} \operatorname{dim} \hat{H}^{-i}\left(Z_{p} ; H^{i}(X ; Z)\right),
$$

where $H^{i}(X ; Z)$ is the $i$ th integral cohomology of $X$. By the universal coefficient theorem, we have $H^{i}(X ; Z) \otimes Z_{p}=\operatorname{Tor}\left(H^{i}(X ; Z) ; Z_{p}\right)=0$ if $0<i<m, m+1<i<n$, $n+1<i<m+n$ or $m+n+1<i$. Hence $H^{-i}\left(Z_{p} ; H^{i}(X ; Z)\right)=0$ for these values of $i$. For other values of $i$, say $i=m$, either $H^{m}(X ; Z) \otimes Z_{p}=Z_{p}$ and $\operatorname{Tor}\left(H^{m}(X ; Z) ; Z_{p}\right)=0$, in which case $\hat{H}^{-m}\left(Z_{p} ; H^{m}(X ; Z)\right)=0$ since $m$ is odd, or else $H^{m+1}(X ; Z) \otimes Z_{p}=0$ and $\operatorname{Tor}\left(H^{m+1}(X ; Z) ; Z_{p}\right)=Z_{p}$, in which case $\hat{H}^{-m-1}\left(Z_{p} ; H^{m+1}(X ; Z)\right)=0$ since $m+1$ is even. In short, using the fact that both $m$ and $n$ are odd, one sees that the above inequality leads to the absurd assertion $3 \leqq 2$.

There are occasions where the last troublesome case $(r=n-m+1, m$ and $n$ are both odd) can be eliminated altogether. For instance if $m=n$. A less trivial case is when the integral cohomology $H^{*}(X ; Z)$ is of finite type (i.e., each $H^{\prime}(X ; Z)$ is finitely generated $)$ - in particular, if $H^{*}(X ; Z)=H^{*}\left(S^{m} \times S^{n} ; Z\right)$. The proof goes as follows. Using integral AWS cochain groups of $X$, one obtains a spectral sequence $E_{r}(X ; Z)$ with

$$
E_{2}^{s, t}(X ; Z)=\hat{H}^{s}\left(Z_{p} ; H^{t}(X ; Z)\right) .
$$

If $H^{*}(X ; Z)$ if of finite type, by (3.8) one sees that $E_{2}^{s, t}(X ; Z)=0$ for all $s$ odd; hence, in particular, $E_{r}^{s, t}(X ; Z)=0$ for $s$ odd and $r \geqq 2$. Now the coefficient homomorphism $\pi: Z \rightarrow Z_{p}$ gives the commutative diagram

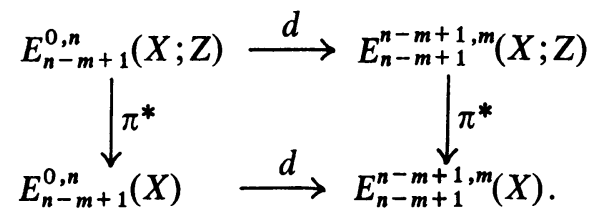

It is not difficult to see that $E_{n-m+1}^{0, n}(X ; Z)=E_{2}^{0, n}(X ; Z)=Z_{p}$ and $\pi^{*}$ on the 
left is an isomorphism. Since $E_{n-m+1}^{n-m+1, m}(X ; Z)=0$ because $n-m+1$ is odd, we have $d=0$ on the bottom or $d_{n-m+1}(1 \otimes b)=0$.

Summarizing all these, we have

THEOREM 3.9. Suppose that $p \neq 2$, that $Z_{p}$ acts trivially on $H^{*}\left(X ; Z_{p}\right)$ and that the spectral sequence $E_{r}(X)$ is nontrivial. Then $H^{*}\left(F ; Z_{p}\right)=H^{*}\left(S^{r} ; Z_{p}\right)$ for some $-1 \leqq r \leqq m+n$ and $r$ is odd. If $m=n$, then $F$ is necessarily empty. If $m \neq n$ and the integral cohomology $H^{*}(X ; Z)$ of $X$ is of finite type, then $F$ can be nonempty only if $m$ is even and $n$ is odd.

For the rest of this section, we assume that the spectral sequence $E_{r}(X)$ is trivial. We have then $\operatorname{dim} H^{*}\left(F ; Z_{p}\right)=4$. Let $0 \leqq m_{1} \leqq n_{1} \leqq l_{1}$ be the dimensions where $H^{*}\left(F ; Z_{p}\right)$ is nonvanishing. By (2.6), we have $m_{1} \leqq m, n_{1} \leqq n$ and $l_{1} \leqq m+n$. First assume that $F$ is connected. Let $a_{1}, b_{1}$ and $c_{1}$ be generators in dimensions $m_{1}>0, n_{1}$ and $l_{1}$ respectively and $1 \in H^{0}\left(F ; Z_{p}\right)$ be the unit of $H^{*}\left(F ; Z_{p}\right)$. The elements $t^{q} \otimes 1, t^{q-m_{1}} \otimes a_{1}, t^{q-n_{1}} \otimes b_{1}$ and $t^{q-l_{1}} \otimes c_{1}$ form a basis of $J^{q}(F)$. We proceed to determine the multiplication in $H^{*}\left(F ; Z_{p}\right)$. Clearly, we have $a_{1} c_{1}=b_{1} c_{1}=c_{1}^{2}=0$.

LEMMA 3.10. The following cases cannot happen:

(1) $a_{1}^{2}=b_{1}^{2}=a_{1} b_{1}=0$.

(2) $a_{1}^{2}=a_{1} b_{1}=0$ and $b_{1}^{2} \neq 0$.

(3) $b_{1}^{2}=a_{1} b_{1}=0$ and $a_{1}^{2} \neq 0$.

Proof. Consider, for example, case (3). Choose some $q$ such that $q-m$ is even. In $J^{q}(X)$, choose $\alpha \in J^{q-m, m}(X)$ representing $t^{q-m} \otimes a$ in $E_{\infty}^{q-m, m}(X)=E_{2}^{q-m, m}(X)$ and $\beta \in J^{q-n, n}(X)$ representing $t^{q-n} \otimes b$ in $E_{\infty}^{q-n, n}(X)=E_{2}^{q-n, n}(X)$. By (2.7), we may assume that

$$
\begin{aligned}
& i^{*}(\alpha)=A t^{q-m_{1}} \otimes a_{1}+B t^{q-n_{1}} \otimes b_{1}+C t^{q-l_{1}} \otimes c_{1}, \\
& i^{*}(\beta)=A^{\prime} t^{q-m_{1}} \otimes a_{1}+B^{\prime} t^{q-n_{1}} \otimes b_{1}+C^{\prime} t^{q-l_{1}} \otimes c_{1},
\end{aligned}
$$

where $A, A^{\prime}, B, B^{\prime}, C, C^{\prime} \in Z_{p}$. Since $q-m$ is even, $\alpha \beta \in J^{2 q-m-n, m+n}(X)$ represents $t^{2 q-m-n} \otimes a b$ in $E_{\infty}^{2 q-m-n, m+n}(X)$. Hence $\alpha \beta \in^{\prime} J^{2 q-m-n+1, m+n-1}(X)$ and, in particular, $\alpha \beta \neq 0$. Now

$$
i^{*}(\alpha \beta)=A A^{\prime} t^{q-m_{1}} \cdot t^{q-m_{1}} \otimes a_{1}^{2} .
$$

It follows that $q-m_{1}$ is even if $p \neq 2, A A^{\prime} \neq 0$, and

$$
t^{2 q-2 m_{1}} \otimes a_{1}^{2}=i^{*}\left(\left(A A^{\prime}\right)^{-1} \alpha \beta\right) \epsilon^{\prime} i^{*}\left(J^{2 q-m-n+1, m+n-1}(X)\right) .
$$

On the other hand, $\alpha^{2} \in J^{2 q-2 m, 2 m}(X)$ represents $t^{2 q-2 m} \otimes a^{2}=0$ in $E_{\infty}^{2 q-2 m, 2 m}(X)$. Hence $\alpha^{2} \in J^{2 q-2 m+1,2 m-1}(X)$ and $t^{2 q-2 m_{1}} \otimes a_{1}^{2}=i^{*}\left(A^{-2} \alpha^{2}\right) \in i^{*}\left(J^{2 q-2 m+1,2 m-1}(X)\right)$. This is a contradiction since $J^{2 q-2 m+1,2 m-1}(X) \subset J^{2 q-m-n+1, m+n-1}(X)$. (1) and (2) can be treated in similar fashion. 
Besides the cases covered by (3.10), there are only five more possibilities. Allowing suitable change of basis in $H^{*}\left(F ; Z_{p}\right)$, it is not difficult to see that one can narrow down to the following three cases:

(4) $a_{1}^{2}=b_{1}^{2}=0$ and $a_{1} b_{1} \neq 0$, i.e., $H^{*}\left(F ; Z_{p}\right)=H^{*}\left(S^{m_{1}} \times S^{n_{1}} ; Z_{p}\right)$ as an algebra. Moreover, $m-m_{1}$ and $n-n_{1}$ are all even if $p \neq 2$.

(5) $a_{1}^{2}=b_{1}, a_{1} b_{1} \neq 0$ and $b_{1}^{2}=0$, i.e., $H^{*}\left(F ; Z_{p}\right)=Z_{p}\left[a_{1}\right] /\left(a_{1}^{4}\right)$ is a truncated polynomial algebra.

(6) $m_{1}=n_{1}$ with $a_{1}^{2} \neq 0, b_{1}^{2} \neq 0$ and $a_{1} b_{1}=0$. Let $b_{1}^{2}=D a_{1}^{2}, D \in Z_{p}$. Using the notations in (3.10), we may take $q=0$ since either $m$ is even or $p=2$. We have $i^{*}(\alpha \beta)=\left(A A^{\prime}+B B^{\prime} D\right) t^{-2 m_{1}} \otimes a_{1}^{2} \neq 0$; hence at least $A$ or $B$ is nonzero. We have $i^{*}\left(\alpha^{2}\right)=\left(A^{2}+B^{2} D\right) t^{-2 m_{1}} \otimes a_{1}^{2}$, hence $i^{*}\left(\alpha^{3}\right)=0$ and therefore $\alpha^{3}=0$. Now $\alpha^{2} \in J^{-2 m+1,2 m-1}(X)=J^{-n, n}(X)$, so we can write $\alpha^{2}=A^{\prime \prime} \beta+B^{\prime \prime} \alpha+C^{\prime \prime}(1 \otimes 1)$. From this we obtain $\alpha^{3}=A^{\prime \prime} \alpha \beta+A^{\prime \prime} B^{\prime \prime} \beta+\left(B^{\prime \prime 2}+C^{\prime \prime}\right) \alpha+B^{\prime \prime} C^{\prime \prime}(1 \otimes 1)=0$. But $\alpha \beta, \beta, \alpha$ and $1 \otimes 1$ form a basis of $J^{0}(X)$. Therefore $A^{\prime \prime}=B^{\prime \prime}=C^{\prime \prime}=0$ or $A^{2}+B^{2} D=0$. This would imply that both $A$ and $B$ are nonzero. If $p=2$, we have $a_{1}^{2}=b_{1}^{2}$. If $p \neq 2$, (6) reduces to (4) by taking $a_{1}+A B^{-1} b_{1}$ and $a_{1}-A B^{-1} b_{1}$ as basis. Thus we obtain the following theorem which generalizes the result of Swan [7, Theorem 6.1].

THEOREM 3.11. Suppose that $Z_{p}$ acts trivially on $H^{*}\left(X ; Z_{p}\right)$, that the spectral sequence $E_{r}(X)$ is trivial and that $F$ is connected. Then

$$
H^{*}\left(F ; Z_{p}\right)=H^{*}\left(S^{m_{1}} \times S^{n_{1}} ; Z_{p}\right)
$$

as a module, where $0<m_{1} \leqq m$ and $m_{1} \leqq n_{1} \leqq n$. The dimension parities $m-m_{1}$ and $n-n_{1}$ are even if $p \neq 2$. The multiplication of $H^{*}\left(F ; Z_{p}\right)$ can be described in one of the following ways:

(3.11.1) $H^{*}\left(F ; Z_{p}\right)=H^{*}\left(S^{m_{1}} \times S^{n_{1}} ; Z_{p}\right)$ as an algebra.

(3.11.2) $n_{1}=2 m_{1}$ and $H^{*}\left(F ; Z_{p}\right)=Z_{p}\left[a_{1}\right] /\left(a_{1}^{4}\right)$ is a truncated polynomial algebra with one generator $a_{1}$ of degree $m_{1}$.

(3.11.3) $p=2, m_{1}=n_{1}$ and generators $a_{1}, b_{1} \in H^{m_{1}}\left(F ; Z_{2}\right)$ can be chosen so that $a_{1}^{2}=b_{1}^{2} \neq 0$ and $a_{1} b_{1}=0$.

The structure of $H^{*}\left(F ; Z_{p}\right)$ when $F$ is disconnected is given as follows, which we state without proof.

THEOREM 3.12. The hypothesis is the same as (3.11) except that $F$ is disconnected. Then $H^{*}\left(F ; Z_{p}\right)$ can be described in one of the following ways:

(3.12.1) $\quad H^{*}\left(F ; Z_{p}\right)=H^{*}\left(S^{m_{1}} \cup S^{n_{1}} ; Z_{p}\right)$, where $0 \leqq m_{1} \leqq n, m_{1} \leqq n_{1} \leqq m+n$ and $\cup$ means disjoint union.

(3.12.2) $\quad F$ has two components $F_{1}$ and $F_{2}$, where $F_{1}$ is acyclic over $Z_{p}$ and

$$
H^{*}\left(F_{2} ; Z_{p}\right)=Z_{p}\left[a_{1}\right] /\left(a_{1}^{3}\right)
$$

is a truncated polynomial algebra with one generator of degree $\leqq n$. 
4. The case where $Z_{p}$ acts nontrivially on $H^{*}\left(X ; Z_{p}\right)$. In this section, we assume that $Z_{p}$ acts nontrivially on $H^{*}\left(X ; Z_{p}\right)$. This is possible, of course, only when $m=n$, which we assume for the rest of this section. We can actually narrow it down further.

Proposition 4.1. If $p \neq 2$ and $n$ is even, then $Z_{p}$ acts trivially on $H^{*}\left(X ; Z_{p}\right)$.

Proof. Let $T$ be a generator of $Z_{p}$ and consider $T^{*}$ on $H^{n}\left(X ; Z_{p}\right)$. Set $T^{*}(a)=A_{11} a+A_{12} b$ and $T^{*}(b)=A_{21} a+A_{22} b$. We have $a b=T^{*}(a b)$ $=A_{11} A_{22} a b+A_{12} A_{21} b a=\left(A_{11} A_{22}+A_{12} A_{21}\right) a b$ since $n$ is even. Hence $A_{11} A_{22}+A_{12} A_{21}=1=A+2 A_{12} A_{21}$, where $A$ is the determinant of $T^{*}$. Since $T^{*}=1$, we have $A^{p}=1$ and hence $A=1$. We obtain therefore that $A_{12} A_{21}=0$ since $p \neq 2$. Similarly, from $T^{*}\left(a^{2}\right)=0$ and $T^{*}\left(b^{2}\right)=0$ we obtain $A_{11} A_{12}=0$ and $A_{21} A_{22}=0$. From these we deduce that $A_{12}=A_{21}=0$ and $A_{11}=A_{22}^{-1}$. But then $T^{* p}=1$ implies that $A_{11}=A_{22}=1$.

If we assume that $H^{*}(X ; Z)$ is of finite type, then it is easily seen that the torsion free part of $H^{n}(X ; Z)$ is $Z+Z$ on which $Z_{p}$ must act trivially if $p>3$. Hence with this additional condition, $Z_{p}$ can act nontrivially on $H^{*}\left(X ; Z_{p}\right)$ only if $p=2$ or $p=3$ and $n$ is odd.

Consider $T^{*}$ on $H^{n}\left(X ; Z_{p}\right)$. As usual, define $\tau=1-T^{*}$ and $\sigma=\Sigma_{0}^{p-1} T^{* i}$. Recall [2, Chapter XII, §7] that

$$
\hat{H}^{s}\left(Z_{p} ; H^{n}\left(X ; Z_{p}\right)\right)=\left\{\begin{array}{l}
\operatorname{ker} \tau / \operatorname{Im} \sigma, s \text { even, } \\
\operatorname{ker} \sigma / \operatorname{Im} \tau, s \text { odd }
\end{array}\right.
$$

THEOREM 4.3. If $p \neq 3$ and $Z_{p}$ acts nontrivially on $H^{*}\left(X ; Z_{p}\right)$, then $H^{*}\left(F ; Z_{p}\right)=H^{*}\left(S^{r} ; Z_{p}\right)$ for some $-1 \leqq r \leqq 2 n$. Moreover, $r$ is odd when $p>3$.

Proof. One sees easily from (4.2) that $\operatorname{dim} E_{2}^{s, n}(X) \leqq 1$. Therefore $\operatorname{dim} H^{*}\left(F ; Z_{p}\right) \leqq 3$. If $p \neq 3$, we must have $\operatorname{dim} H^{*}\left(F ; Z_{p}\right)=0$ or 2 in view of (2.8). Notice that (4.3) is true even if $H^{*}\left(X ; Z_{p}\right)=H^{*}\left(S^{n} \times S^{n} ; Z_{p}\right)$ as a module only.

Consider now the case $p=3$. Since $T^{*}$ satisfies $x^{3}-1=(x-1)^{3}=0$ but not $x-1=0$, the minimal polynomial of $T^{*}$ is $(x-1)^{2}=x^{2}+x+1$. Therefore 1 is an eigenvalue of $T^{*}$ whose eigenspace has dimension 1 . In other words, we have $\operatorname{dim} \operatorname{ker} \tau=1$ and $\operatorname{dim} \operatorname{ker} \sigma=2$. By (4.2), this gives $\operatorname{dim} E_{2}^{s, n}(X)=1$ for all $s$. Moreover, generators $a, b \in H^{n}\left(X ; Z_{3}\right)$ can be so chosen that we have $a^{2}=b^{2}=0, a b \neq 0, T^{*}(a)=a$ and $T^{*}(b)=a+b$. Now $E_{2}(X)$ can be described as follows. For $s$ even, a generator $\alpha^{s} \in E_{2}^{s, n}(X)$ is represented by $a \in H^{n}\left(X ; Z_{3}\right)$. For $s$ odd, a generator $\beta^{s} \in E_{2}^{s, n}(X)$ is represented by $b \in H^{n}\left(X ; Z_{3}\right)$. The generators of $E_{2}^{s, 0}(X)$ and $E_{2}^{s, 2 n}(X)$ are still denoted by $t^{s} \otimes 1$ and $t^{s} \otimes a b$ respectively. According to [2, Chapter XII, §7], the multiplication in $E_{2}(X)$ is given by 


$$
\begin{gathered}
\left(t^{s} \otimes 1\right) \alpha^{t}=\alpha^{t}\left(t^{s} \otimes 1\right)= \begin{cases}0, & s \text { odd }, \\
\alpha^{s+t}, & s \text { even, }\end{cases} \\
\left(t^{s} \otimes 1\right) \beta^{t}=\beta^{t}\left(t^{s} \otimes 1\right)= \begin{cases}\beta^{s+t}, & s \text { even, } \\
-\alpha^{s+t}, & \text { s odd },\end{cases} \\
\alpha^{s} \alpha^{t}=\alpha^{t} \alpha^{s}=0, \\
\alpha^{s} \beta^{t}=-\beta^{t} \alpha^{s}=t^{s+t} \otimes a b, \\
\beta^{s} \beta^{t}=\beta^{t} \beta^{s}=t^{s+t} \otimes b a .
\end{gathered}
$$

LEMMA 4.5. $\operatorname{dim} H^{*}\left(F ; Z_{3}\right) \neq 2$.

Proof. The only possible nontrivial differentials in $E_{r}(X)$ are $d_{n+1}$ and $d_{2 n+1}$. Therefore we can always identify $E_{2}(X)$ with $E_{n+1}(X)$. If $d_{n+1}=0$, one sees from (4.4) that $E_{r}(X)$ must be trivial. In this case, we have $\operatorname{dim} H^{*}\left(F ; Z_{3}\right)=3$. Suppose $d_{n+1}$ is nontrivial. From (4.4) again we must have $d_{n+1}\left(\beta^{1}\right) \neq 0$ and we may set $d_{n+1}\left(\beta^{1}\right)=t^{n+2} \otimes 1$. Remember that $n$ is odd; using (4.4) once more we compute that

$$
d_{n+1}\left(\beta^{-n}\right)=d_{n+1}\left[\left(t^{-n-1} \otimes 1\right) \beta^{1}\right]=\left(t^{-n-1} \otimes 1\right)\left(t^{n+2} \otimes 1\right)=t \otimes 1 \neq 0
$$

and

$$
d_{n+1}\left(\beta^{-n} \beta^{1}\right)=(t \otimes 1) \beta^{1}+\beta^{-n}\left(t^{n+2} \otimes 1\right)=-\alpha^{2}-\alpha^{2}=\alpha^{2} \neq 0 .
$$

These relations imply that $E_{n+2}^{1, n}(X)=E_{n+2}^{-n+1,2 n}(X)=0$. Hence

$$
\operatorname{dim} H^{*}\left(F ; Z_{3}\right)=\operatorname{dim} J^{n+1}(X) \leqq 1 .
$$

Because $n$ is odd, we know $\operatorname{dim} H^{*}\left(F ; Z_{3}\right) \neq 1$. Hence either $F=\varnothing$ or $\operatorname{dim} H^{*}\left(F ; Z_{3}\right)=3$. The structure of $H^{*}\left(F ; Z_{3}\right)$ is trivial if $F$ is disconnected. Indeed, that must be the case.

LEMMA 4.6. If $\operatorname{dim} H^{*}\left(F ; Z_{3}\right)=3$, then $F$ cannot be connected.

Proof. The spectral sequence $E_{r}(X)$ is trivial. Let $a_{1}, b_{1} \in H^{*}\left(F ; Z_{3}\right)$ be generators of positive dimensions $m_{1}$ and $n_{1}$ respectively, where $0<m_{1} \leqq n_{1}$. Both $m_{1}$ and $n_{1}$ are even by (2.8). Clearly, $a_{1} b_{1}=b_{1}^{2}=0$. We may choose $\bar{\beta} \in J^{-n, n}(X)$ representing $\beta^{-n}$ in $E_{2}^{-n, n}(X)$ and

$$
i^{*}(\bar{\beta})=A t^{-m_{1}} \otimes a_{1}+B t^{-n_{1}} \otimes b_{1} .
$$

Similarly, we may choose $\bar{\alpha} \in J^{-n+1, n}(X)$ representing $\alpha^{-n+1}$ in $E_{2}^{-n+1, n}(X)$ and

$$
i^{*}(\bar{\alpha})=A^{\prime} t^{-m_{1}+1} \otimes a_{1}+B^{\prime} t^{-n_{1}+1} \otimes b_{1} .
$$

We have $i^{*}\left(\beta^{2}(t \otimes 1)\right)=A^{2} t^{-2 m_{1}+1} \otimes a_{1}^{2}$. Since $\bar{\beta}^{2}(t \otimes 1) \in J^{-2 n+1,2 n}(X)$ repre- 
sents $0 \neq\left(t^{-2 n} \otimes 1\right)(t \otimes 1)$ in $E_{2}^{-2 n+1,2 n}(X)$, we have $A \neq 0, \quad a_{1}^{2} \neq 0$ and $0 \neq t^{-2 m_{1}+1} \otimes a_{1}^{2} \epsilon^{\prime} i^{*}\left(J^{-2 n+2,2 n-1}(X)\right)$. Similarly, we have

$$
i^{*}(\bar{\alpha} \bar{\beta})=i^{*}(\bar{\beta} \bar{\alpha})=A A^{\prime} t^{-2 m_{1}+1} \otimes a_{1}^{2} .
$$

Since $\bar{\alpha} \bar{\beta} \in J^{-2 n+1,2 n}(X)$ represents $0 \neq t^{-2 n+1} \otimes a b$ in $E_{2}^{-2 n+12 n}(X)$, we have $A^{\prime} \neq 0$. Now by (4.4), $\alpha^{-n+1} \beta^{-n}=-\beta^{-n} \alpha^{-n+1}$. Hence $2 \bar{\alpha} \bar{\beta} \in J^{-2 n+1,2 n}(X)$ represents 0 in $E_{2}^{-2 n+1,2 n}(X)$. In other words, $2 \bar{\alpha} \bar{\beta} \in J^{-2 n+2,2 n-1}(X)$. Since $A, A^{\prime} \neq 0$ and $p \neq 2$, this means $t^{-2 m_{1}+1} \otimes a_{1}^{2} \in i^{*}\left(J^{-2 n+2,2 n-1}(X)\right)$, a contradiction.

If we assume that $H^{*}(X ; Z)$ is of finite type, then the $E_{2}$-term of the integral spectral sequence $E_{r}(X ; Z)$ can easily be computed. Comparing $E_{r}(X)$ with $E_{r}(X ; Z)$ as we have done before, one sees that $E_{r}(X)$ is trivial. Hence in this case we have $F \neq \varnothing$. Summing up, we have therefore proved

THEOREM 4.7. If $p=3$ and $Z_{3}$ acts nontrivially on $H^{*}\left(X ; Z_{3}\right)$, then $F$ is either empty or it has two components $F_{1}$ and $F_{2}$, where $F_{1}$ is acyclic over $Z_{3}$ and $H^{*}\left(F_{2} ; Z_{3}\right)=H^{*}\left(S^{2 r} ; Z_{3}\right)$ for some $0 \leqq r \leqq n$. Moreover, if the integral cohomology $H^{*}(X ; Z)$ of $X$ is of finite type, then $F$ is necessarily nonempty.

5. Some examples. We give in this section a few examples to demonstrate the various results of the last two sections. These examples, however, do not exhaust all the possibilities permitted by the theorems we obtained. Hence the content of this section is rather incomplete.

(3.2) and (3.9). Let $f: S^{3} \rightarrow S^{2}$ be the Hopf map. Let $X$ be the union of the mapping cylinders $S^{3} \times S^{r} \rightarrow S^{2} \times S^{r}$ and $S^{3} \times S^{r} \rightarrow S^{3}$. Then $H^{*}(X ; Z)$ $=H^{*}\left(S^{2} \times S^{r+2} ; Z\right)$. Let $Z_{p}$ act freely on $S^{r}(r$ odd if $p \neq 2)$ and trivially on $S^{3}$ and $S^{2}$. This defines an action of $Z_{p}$ on $X$ for which $F=S^{3}$. Similar examples can be constructed using other Hopf maps.

For Theorems (3.11) and (3.12), we shall only give examples for the case $p=2$. (3.11.1) is of course trivial.

(3.11.2) Let SU(3) be the special unitary 3-group. It is known [1, Proposition 9.1] that $H^{*}(\mathrm{SU}(3) ; Z)=H^{*}\left(S^{3} \times S^{5} ; Z\right)$. Let $Z_{2}$ act on $\mathrm{SU}(3)$ by conjugation. Then $F=\mathrm{SO}(3)$, the special orthogonal group which is topologically a real projective 3-space.

(3.11.3) Consider the Klein bottle $F$ as the nontrivial $S^{1}$ bundle over $S^{1}$. Then $H^{*}\left(F ; Z_{2}\right)$ is that described by (3.11.3). Let $\pi: S^{1} \rightarrow S^{1}$ be the doudle covering map and $X$ be the join of $F$ with this $S^{0}$ bundle. Then $X=S^{1} \times S^{2}$. The natural involution of $\pi$ carries over to an involution on $X$ with $F$ as fixed point set.

(3.12.1) Let $X=\mathrm{U}(2)$ be the unitary 2-group. Then [1], $H^{*}(X ; Z)=H^{*}\left(S^{1} \times S^{3} ; Z\right)$. The conjugation is an involution on $X$ with $F=\mathrm{O}(2)$, the crthogonal 2-group which is two copies of $S^{1}$. Similarly, $x \rightarrow x^{-1}$ is an involution on $X$ whose fixed 
point set consists of two isolated points $x=1, x=-1$, and a $S^{2}$ formed by those $x \in \mathrm{U}(2)$ of the form

$$
x=\left(\begin{array}{c}
a, z \\
\bar{z},-a
\end{array}\right), a \text { real. }
$$

One can also take $X=S^{1} \times S^{2}$, thinking $S^{1}$ as imbedded in $S^{2}$ as the equator. For $x \in S^{1}$, let $T_{x}$ be the rotation of $S^{2}$ of an angle $2 \pi / p$ with $x$ as axis. Then $(x, y) \rightarrow\left(x, T_{x}(y)\right)$ defines an action of $Z_{p}$ on $X$ with $F=S^{1} \cup S^{1}$.

(3.12.2) On SU(3), $x \rightarrow x^{-1}$ is an involution whose fixed point set can be seen to be the disjoint union of the point $x=1$ and a complex projective plane formed by those $x \in \mathrm{SU}(3)$ having a complex line as invariant subspace.

(4.3) The simplest case is letting $Z_{2}$ act on $S^{n} \times S^{n}$ by interchanging coordinates. We can also consider the double covering $\pi: S^{1} \rightarrow S^{1}$ and take $X$ to be the union of two copies of the mapping cylinders of $\pi$. Then $X$ is just the Klein bottle so that $H^{*}\left(X ; Z_{2}\right)=H^{*}\left(S^{1} \times S^{1} ; Z_{2}\right)$ additively. Interchanging the mapping cylinders is an involution of $X$ with nontrivial action on $H^{*}\left(X ; Z_{2}\right)$. The fixed point set is $S^{1}$. Similar examples can be constructed using Hopf maps.

(4.7) On $X=S^{1} \times S^{1}$, the map $(x, y) \rightarrow\left(y, x^{-1} y^{-1}\right)$ defines an action of $Z_{3}$ with nontrivial action on $H^{*}\left(X ; Z_{3}\right)$. The fixed point set is three isolated points. Letting $x, y$ be quaternions and Cayley numbers, we get actions of $Z_{3}$ on $S^{3} \times S^{3}$ and $S^{7} \times S^{7}$ respectively. The fixed point set amounts to solving the equation $x^{3}=1$ in these algebras. For the quaternion, it is a point and a 2-sphere. For the Cayley number, it is a point and a 6-sphere.

6. The Poincaré duality. As mentioned in the introduction, our results can be interpreted in terms of Poincaré duality. We say a space $X$ satisfies the $\bmod p$ Poincaré duality if (i) $X$ is connected compact Hausdorff, (ii) there is an integer $n \geqq 0$ such that $H^{k}\left(X ; Z_{p}\right)=0$ for all $k>n$ and $H^{n}\left(X ; Z_{p}\right)=Z_{p}$, (iii) for any $0 \leqq k \leqq n$, the cup-product pairing $H^{k}\left(X ; Z_{p}\right) \otimes H^{n-k}\left(X ; Z_{p}\right) \rightarrow H^{n}\left(X ; Z_{p}\right)$ is nonsingular. Consider the following conjecture.

CONJECTURe. If $Z_{p}$ acts on a space $X$ satisfying the $\bmod p$ Poincaré duality, then each component of the fixed point set also satisfies the $\bmod p$ Poincaré duality.

The simplest case is when $X$ is a (cohomology) sphere; there the conjecture is true by the theorem of P. A. Smith. A result of the author [6] says that the conjecture is also true when $X$ is a (cohomology) real projective space or a (cohomology) lens space. Our present result can be summarized by saying that the conjecture is again true when $X$ is a product of two spheres. This can be seen simply by checking from case to case. The validity of the above conjecture in general might be a problem of some interest. 


\section{REFERENCES}

1 A. Borel, Sur la cohomologie des espaces fibrés principaux et des espaces homogènes de groupes de Lie compacts, Ann. of Math. (2) 57 (1953), 115-207.

2. H. Cartan and S. Eilenberg, Homological algebra, Princeton Univ. Press, Princeton, N.J., 1956.

3. E. E. Floyd, On periodic maps and the Euler characteristic of associated spaces, Trans. Amer. Math. Soc. 72 (1952), 138-147.

4. P. A. Smith, Fixed points of periodic transformations, Appendix B of S. Lefschetz. Algebraic topology, Amer. Math. Soc. Colloq. Publ. Vol. 27, Amer. Math. Soc., Providence, R. I., 1942.

5. J. C. Su, A note on periodic transformations, Hung-Ching Chow Sixtieth Anniversary Volume, pp. 99-107, Academia Sinica, Taiwan, 1962.

6. - Transformation groups on cohomology projective spaces, Trans. Amer. Math. Soc. 106 (1963), 305-318.

7. R. G. Swan, A new method in fixed point theory, Comment. Math. Helv. 34 (1960), 1-16.

\section{UNIVERSITY OF VIRGINIA,}

Charlottesville, Virginia 